Classification

Physics Abstracts

$05.70 \mathrm{~L}-82.70-81.35-82.20 \mathrm{M}$

\title{
Maximum Entropy Analysis of Disordered Droplet Patterns
}

\author{
Clément Sire $\left(^{*}\right)$ and Michael Seul \\ AT\&T Bell Laboratories, Murray Hill, NJ 07974, U.S.A.
}

(Received 6 June 1994, received in final form 12 September 1994, accepted 16 September 1994)

Résumé. - Nous développons un formalisme d'entropie maximale afin de décrire les configurations désordonnées formées au cours de la séparation de phase d'un mélange binaire bidimensionnel. Nous montrons que les corrélations provenant des contraintes géométriques locales conduisent à une distribution quasiment gaussienne pour les rayons des bulles de la phase minoritaire. L'examen statistique des propriétés géométriques et topologiques des images expérimentales révèle le rôle majeur joué par l'écrantage à courte distance de la charge topologique, et des fluctuations de la taille des bulles.

Abstract. - A maximum entropy description is developed for disordered planar patterns of droplets and applied to the analysis of such patterns formed in a two-dimensional binary mixture during late-stage coarsening. Global correlations, arising from local "packing" constraints, are shown to produce a virtually symmetric shape of the scaled droplet radius distribution. The examination of geometrical and topological pattern statistics reveals a prominent role of screening at short distance, both of domain area fluctuations, as well as of topological charge.

\section{Introduction}

Polydisperse assemblies of droplets are observed in a variety of experimental situations, notably including film deposition from a vapor phase [1,2], "packing" in granular materials [3], as well as late-stage coarsening, or "Ostwald ripening", of minority phase domains initiated by a rapid temperature or field quench [4]. Significant polydispersity generally precludes spatial correlations of long range, even in systems governed by competing interactions which favor the ordering of domains into modulated phases [5-9]. To describe such globally disordered structures, one may elucidate the nature of the short-range correlations, a classic topic of interest in the characterization of amorphous phases [10]. In addition, one may determine the interdependence of topological and geometrical quantities, notably the joint probability distribution $P(n, A)$, of local droplet coordination $n$ and droplet volume or area, $A$ : this function is essential to the description of cellular structures [11].

(*) Permanent Address: Laboratoire de Physique Quantique, Université Paul Sabatier, 31062 Toulouse, Cedex, France. E-mail: Clement@siberia.ups-tlse.Fr 
Direct imaging methods facilitate the evaluation of these quantities in two and, increasingly, in three dimensions. This approach has been recently employed to investigate spatial correlations in planar, disordered patterns of coarsening domains, formed in a two-dimensional binary mixture of amphiphilic molecules subsequent to a rapid (surface) pressure quench [12]. Motivated by these experiments, we have developed a maximum entropy analysis of disordered droplet patterns. The theory is a generalization of the maximum entropy analysis of cellular patterns, originated by Rivier [13]. It is predicated upon the existence of a partition of space with the property that each droplet is contained within a (polyhedral) cell of the partition. In addition, we introduce a constraint in the form of presetting the second moment, $\mu_{2}$, of the distribution of droplet coordination numbers. The theory yields predictions for the joint probability distribution $P(n, A)$, for the droplet radius distribution, and for the spatial correlations between areas of adjacent droplets.

Following a summary of theoretical results in Section 2, we apply the theory, in Section 3, to account for the near-Gaussian shape of the droplet radius distribution and for short-range screening of topological charge in coarsening droplet domain patterns in the experimental system of interest here, namely a binary mixture of amphiphiles forming a monomolecular Langmuir film at an air-water interface. In Section 4, we address the essential assumptions of maximum entropy analysis in relation to the aforementioned two-dimensional binary mixture in the process of late-stage coarsening, the latter a subject of extensive theoretical as well as experimental interest $[4,14]$. We end by discussing the role of entropy maximization in defining structural aspects of other systems.

\section{Maximum Entropy Analysis of Droplet Patterns}

2.1. Preliminaries. - In the following, we develop a maximum entropy (ME) description [15] of disordered droplet patterns. This approach was introduced by Rivier and coworkers in the context of cellular patterns such as those formed by soap froths [13]. It is based on the assumption that pattern configurations are selected to satisfy general geometrical constraints rather than energetic constraints, which, if present, give rise only to correlations of short range. In particular, these energetic constraints must not restrict or bias access to any pattern configurations.

We are interested here in planar patterns formed by a polydisperse set of circular bubbles which occupy a fraction $c$ of the available area. A typical example is shown in Figure 1. Patterns are disordered in the sense that the pair correlation is essentially that of a liquid. To perform a statistical analysis of the local pattern structure, we assign to each bubble configuration a partition of space (here: total sample area). Such a partition is provided by the Voronoi diagram of bubble centroids [16]. If the area fraction, $c$, is sufficiently small, and the bubble pattern is locally "relaxed" [17], one generally finds, as in Figure 1, that each bubble is embedded entirely in the Voronoi polygon containing the bubble centroid [18]. The application of the ME formalism to space filling cellular patterns, leading to Lewis or Aboav law is not new. However, the generalization to non space filling patterns (here bubble patterns), the quantitative description of them, and especially the study of correlations of bubble areas (see Sect. 3.3) are definitely original.

In the presence of predominantly geometrical constraints we expect a maximum entropy analysis to yield the correct description for the cellular pattern, formed by the Voronoi diagram. Specifically, this would imply the description to conform to Lewis [11] and Aboav-Weaire [11] laws, to be discussed in detail below, as well as to reproduce the correct form of the distribution of Voronoi cell areas. In addition, however, we will be interested in the collective properties of the bubbles themselves, notably the nature and range of spatial correlations and the form of 


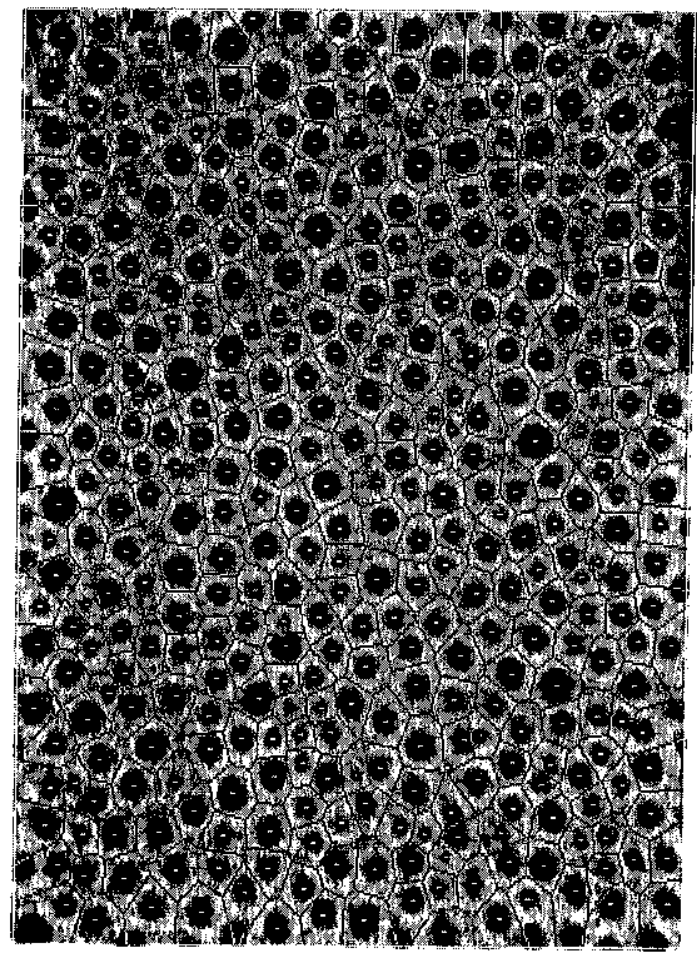

Fig. 1. - Droplet pattern with superimposed Voronoi diagram. Snapshots of a flat-fielded domain pattern recorded during coarsening of a two-dimensional binary mixture, composed of the phospholipid dimyristoylphosphatidylcholine (DMPC) and dihydrocholesterol (dCh) and confined to an air-water interface in the form of a monomolecular (Langmuir) film [12]. The image was recorded after 1249 min of coarsening, following a surface-pressure quench of an off-critical mixture, with mole fraction of $\mathrm{dCh}$ of 0.2 , at $19^{\circ} \mathrm{C}$. Superimposed is the corresponding Voronoi diagram of bubble centroids which are also indicated. The horizontal dimension of the field of view is $1500 \mu \mathrm{m}$.

the droplet radius distribution.

Within the context of late-stage coarsening, the classic Lifshitz-Slyozov (LS) theory [19] gives an explicit solution for this distribution in the limit of dilute bubble patterns. In this regime, geometrical constraints will not affect the radius distribution of very distant droplets, so that maximum entropy considerations apply solely to the Voronoi pattern. However, for moderate area fraction $(0.2 \lesssim c \lesssim 0.3$ ), geometrical ("packing") constraints for the bubble pattern predominate. That is, geometrical constraints which apply to the Voronoi cell areas are transfered to the bubble areas whenever bubbles occupy a non-negligible fraction of their associated Voronoi polygons. This will become clear below. As a consequence, we expect important deviations from the LS theory, and it is these we account for on the basis of ME theory.

2.2. Maximum Entropy Model. - We now present the main steps of the ME calculation. To describe the scaling regime of the droplet coarsening dynamics, we define $P_{n}(a, x)$ as the probability of finding a bubble of area $x\langle A\rangle$ in an $n$-sided Voronoi cell of area $a\langle A\rangle$. Here, $\langle A\rangle$ denotes the average droplet area which evolves in time according to a growth law of the form $\langle A\rangle \sim t^{2 \alpha}$. The coarsening experiments [12] of two-dimensional droplet patterns whose 
analysis we address in Section 3 yield $\alpha \simeq 0.28$. Measuring $a$ and $x$ in units of $\langle A\rangle$, one has $\langle a\rangle=1$ and $\langle x\rangle=c$, where $c$ represents the volume fraction of minority phase; $p_{n}, a_{n}, x_{n}$ respectively denote the density of bubbles with $n$ neighbors (and thus an $n$-sided associated Voronoi polygon), and the averages of $a$ and $x$ for these bubbles. We define the entropy of a given configuration in terms of $P_{n}(a, x)$ in the usual form [11]

$$
S=-\int \mathrm{d} a \mathrm{~d} x P_{n}(a, x) \ln \left(P_{n}(a, x) / P_{0}(a, x)\right)
$$

where $P_{0}(a, x) \sim(a x)^{d}$ captures the fact that, in dimension $d$, one needs at least $(d+1)$ points to define a cell. We now seek to determine the joint distribution $P_{n}(a, x)$ so as to maximize $S$, taking into account all constraints to be satisfied in the course of pattern evolution. These constraints read

$$
\begin{gathered}
\sum_{n \geq 3} p_{n}=1, \sum_{n \geq 3} p_{n} a_{n}=1, \sum_{n \geq 3} p_{n} x_{n}=c \\
\sum_{n \geq 3} p_{n}(n-6)=0
\end{gathered}
$$

The three first conditions, equation (2), respectively ensure: proper normalization of probability, selection of an area-filling froth structure, and conservation of (minority phase) matter. The second condition has the form appropriate for two-dimensional patterns. Equation (3) states the Euler-Poincaré theorem: applied to a pattern of 3 -fold vertices, it requires the average number of neighbors of a cell to be six; defining $C \equiv n-6$ to denote the topological charge of an $n$-fold coordinated bubble, we recognize equation (3) to represent the condition of global charge neutrality, $\langle C\rangle=0$.

To describe a relaxed bubble pattern, we apply the additional constraint to preset the second moment of the distribution $p_{n}$ :

$$
\sum_{n \geq 3} p_{n}(n-6)^{2}=\mu_{2}
$$

Following Lauritsen et al. [20], who have recently studied relaxed Voronoi patterns, with polygon energy $E_{n}=(n-6)^{2}$, in a thermal bath, we recognize this constraint to describe conservation of energy. Since the constraint (4) will be linearly coupled to the entropy (1) via a Lagrange multiplier, entropy maximization is equivalent to minimization of an effective free energy $F=\mu_{2}-\hat{T} S$. Presetting $\mu_{2}$ thus corresponds to setting an effective temperature, $\hat{T}$, which controls the relative importance of entropy and local energetic constraints. In the coarsening experiments of interest here, $\mu_{2}$ generally falls in the range $0.65 \lesssim \mu_{2} \lesssim 0.85$.

A final constraint is required to take into account the fact that each Voronoi cell contains exactly one bubble: accordingly, we limit the area of a bubble so as not to exceed that of its associated Voronoi cell. For a bubble with $n$ neighbors, this condition reads $x \leq \alpha_{n} a ; \alpha_{n}<1$ is a geometrical factor which, for circular bubbles, has the form $(2 \pi / n)^{-1} \sin (2 \pi / n)$, assuming the "average" $n$-sided Voronoi cell to be a regular polygon. Our results do not depend in any essential way on the precise form of $\alpha_{n} \sim 1$; however, it should be noted that the condition $\alpha_{n}<1$ contributes to an increase in the effective area fraction, $c$. In the following, we will take $\alpha_{n}=(2 \pi / n)^{-1} \sin (2 \pi / n)$, or more simply $\alpha_{n}=1$, with the understanding that the effective $c$ in (2) slightly exceeds the nominal experimental value. We shall see below that repulsive interactions between droplets increase even more the effective area fraction to an estimated $0.35 \lesssim c_{\text {eff }} \lesssim 0.5$ for an experimental $c \approx 0.25$. 
Introducing a Lagrange multiplier for each constraint and taking the functional derivative of $S$, one finds

$$
P_{n}(a, x)=\theta\left(\alpha_{n} a-x\right) \frac{(a x)^{2}}{Z} \exp -\left(\lambda_{n}^{(a)} a+\lambda_{n}^{(x)} x+\lambda_{1}(n-6)+\lambda_{2}(n-6)^{2}\right)
$$

where $\theta$ is the step function, and the different Lagrange multipliers $\left(Z, \lambda_{n}^{(a)}, \lambda_{n}^{(x)}, \lambda_{1}, \lambda_{2}\right)$, to be determined according to the above constraints, are functions of $a_{n}, x_{n}, c$ and $\mu_{2}$. As first proposed by Rivier and coworkers [13], we now maximize the entropy as a function of the $a_{n}$ 's and $x_{n}$ 's only, since $\mu_{2}$ and $c$ are preset so as to match experiment. This is achieved by relaxing the constraints on $P_{n}(a, x)$ : accordingly, the $a_{n}$ 's and $x_{n}$ 's are chosen such that the pertinent conditions in equations (2)-(4) are no longer mutually independent. This gives

$$
\begin{aligned}
& a_{n}=1+\lambda_{a}(n-6)+\nu_{a}\left((n-6)^{2}-\mu_{2}\right) \\
& x_{n}=c\left(1+\lambda_{x}(n-6)+\nu_{x}\left((n-6)^{2}-\mu_{2}\right)\right)
\end{aligned}
$$

In a physical structure for which cells experience strong energetic constraints (e.g. in the form of surface tension for soap bubbles), the quadratic term in the expression for $a_{n}$ is expected to dominate. This would lead to the "radius law", $r_{n} \sim a_{n}^{1 / 2} \sim n-n_{0}$, which is actually observed in such systems [11]. In the experiments on coarsening droplet patterns, $\nu_{a}$ and $\nu_{x}$ are expected to be small and their contribution is negligible in the range $4 \leq n \leq 8$ for which the distribution $p_{n}$ is non-zero. As a result, equations (6) and (7) reduce to the so-called Lewis law, $a_{n}=1+\lambda_{a}(n-6)$ and $x_{n}=c\left(1+\lambda_{x}(n-6)\right)$, known to hold in a variety of cellular patterns [11].

The Lewis law analysis of coarsening droplet patterns, illustrated in Figure 2, yields typical values of $\lambda_{a} \approx 0.15$ and $\lambda_{x} \approx 0.23$. We find it useful to think of the parameter $\lambda_{x}$ as a susceptibility which determines the magnitude of the geometrical response, i.e. a change in the average bubble area, to variations of a topological quantity, i.e. a change in coordination number. As geometrical (packing) constraints are relaxed at lower area fraction, the susceptibility is expected to decrease. The absence of geometrical constraints in the dilute limit would imply a vanishing susceptibility: in that limit, the droplet radius distribution would be independent of coordination number, in accordance with LS theory. In contrast, Voronoi polygons are always subject to the geometrical constraint to form a space-filling structure. The precise values of $\lambda_{a, x}$ are immaterial for our subsequent results, and for convenience, we retain the values found here for the remainder of the article.

Having obtained the $a_{n}$ 's and $x_{n}$ 's, one determines $P_{n}(a, x)$ and $p_{n}$ after integrating over $a$ and $x$. In particular, one essentially has $\lambda_{n}^{(a)} \propto 1 / a_{n}$ and $\lambda_{n}^{(x)} \propto 1 / x_{n}$. Our distribution $p_{n}$ is almost independent of $\lambda_{a}$ and $\lambda_{x}$, but is of course sensitive to the value of $\mu_{2}$ which we take to be that found experimentally. Both distributions $p_{n}$ are then very similar, with $p(6) \approx 0.45$, $p(2) \simeq p(5) \approx 0.25$ and $p(4) \simeq p(8) \ll p(6)$.

\section{Results for Coarsening Droplet Patterns}

3.1. Correlation between Droplet and Voronoi Polygon Areas . - We are now in position to examine more closely the relation between $a$ and $x$ for a given bubble. Let $\bar{x}(a)$ represent the average area of bubbles found in Voronoi cells of area $a$. For very small area fraction, $x$ and $a$ are essentially uncorrelated, and thus $\bar{x}(a) \approx c$, independent of $a$. While Lewis's law is expected to remain valid in this regime for the Voronoi pattern, the absence 

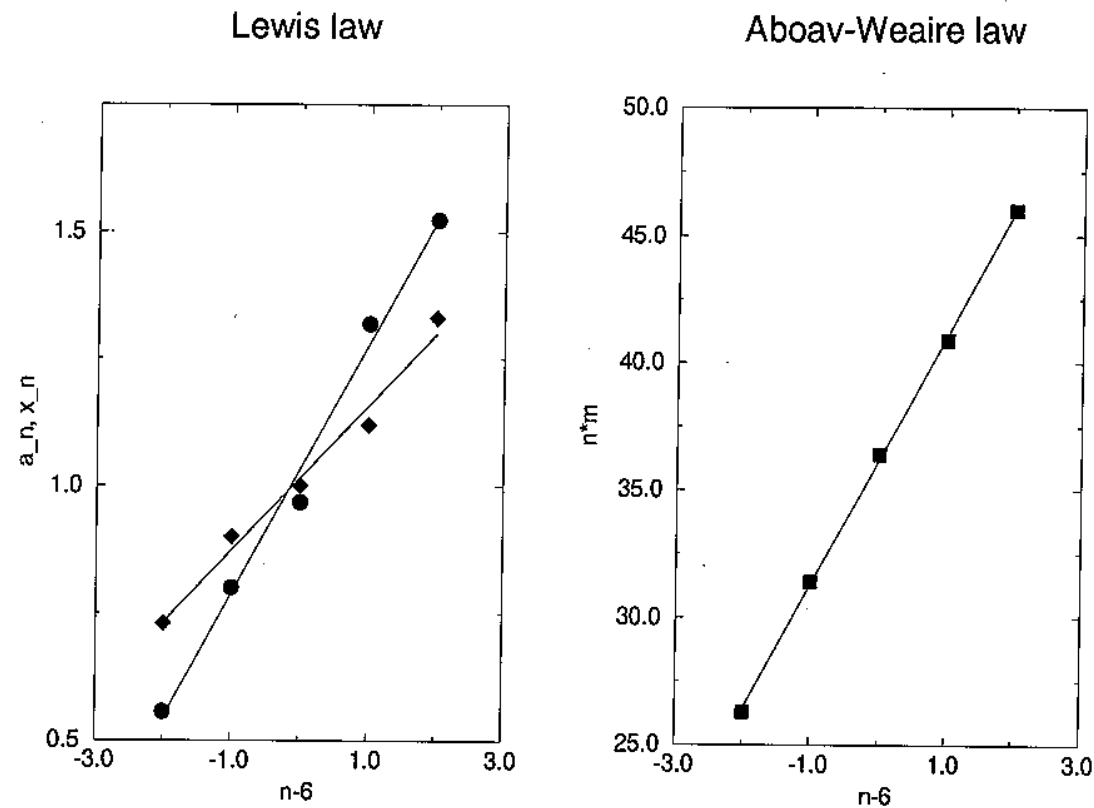

Fig. 2. - Geometrical and topological statistics. Plots (with linear fits) corresponding to Lewis law for droplet pattern (solid circle) and corresponding Voronoi diagram (diamond) (left) and Aboav-Weaire law (right), as discussed in the text. Here, $x_{n} \equiv\left\langle A_{n}\right\rangle /\langle A\rangle$ and $a_{n}=\left\langle A_{V p}\right\rangle_{n} /\left\langle A_{V p}\right\rangle$, respectively represent the average area of an $n$-fold coordinated droplet, normalized by the mean droplet area, $\langle A\rangle$, and the average area of an $n$-sided Voronoi cell, normalized by the mean cell area, $\left\langle A_{V p}\right\rangle ; n$ and $m$ respectively denote: the droplet coordination number and the average coordination number of droplets in the NN shell of an $n$-fold droplet.

of geometrical constraints for bubble areas implies $\lambda_{x} \approx 0$. For coarsening droplet patterns, the distribution of areas is now described by LS theory; however, as a theory applicable to an isolated bubble, LS theory has nothing to say about the structure of the Voronoi pattern which continues to be described by ME theory. For larger volume fraction, typical of that of the experiments of interest here, one finds $\bar{x}(a) \approx c a$, for $a \leq 1$; then, given a finite area faction, $\bar{x}(a)$ saturates at a value larger than $c$.

The plot of $\bar{x}(a)$ vs. $a$ in Figure 3, constructed for the experimental pattern in Figure 1, reveals a strong correlation between bubble and cell areas. While statistics are very poor for small and large $a$ (in accordance with the form of the respective distributions, see below), the correlation is roughly linear. The non-zero intercept of the linear fit, $\bar{x}(a) / c \approx 1.5 a-0.5$, reflects an excluded volume effect: rather than the nominal area fraction $\bar{x}$ it is the quantity $x_{\text {eff }} \approx \bar{x}+0.5 c$ which exhibits a linear dependence on $a$ with vanishing intercept. Consequently, the effective area fraction in fact substantially exceeds, by roughly $50 \%$, the nominal value. We attribute this excluded volume effect to repulsive interactions between adjacent bubbles $[21 ; 22]$. The dependence of the individual (non-averaged) $x(a)$ on $a$ is better described by $x(a) / c \approx 2.5 a-1.5$. Averaging this relation over $n$-sided bubbles, and comparing with the Lewis law, we find that the condition $\bar{x}(a=1) / c=1$ should hold, in accordance with what is observed (see Fig. 3), and that $\lambda_{x}=1.5 \lambda_{a}$, in agreement with the experimental values $\lambda_{\alpha} \approx 0.15$ and $\lambda_{x} \approx 0.23$. 

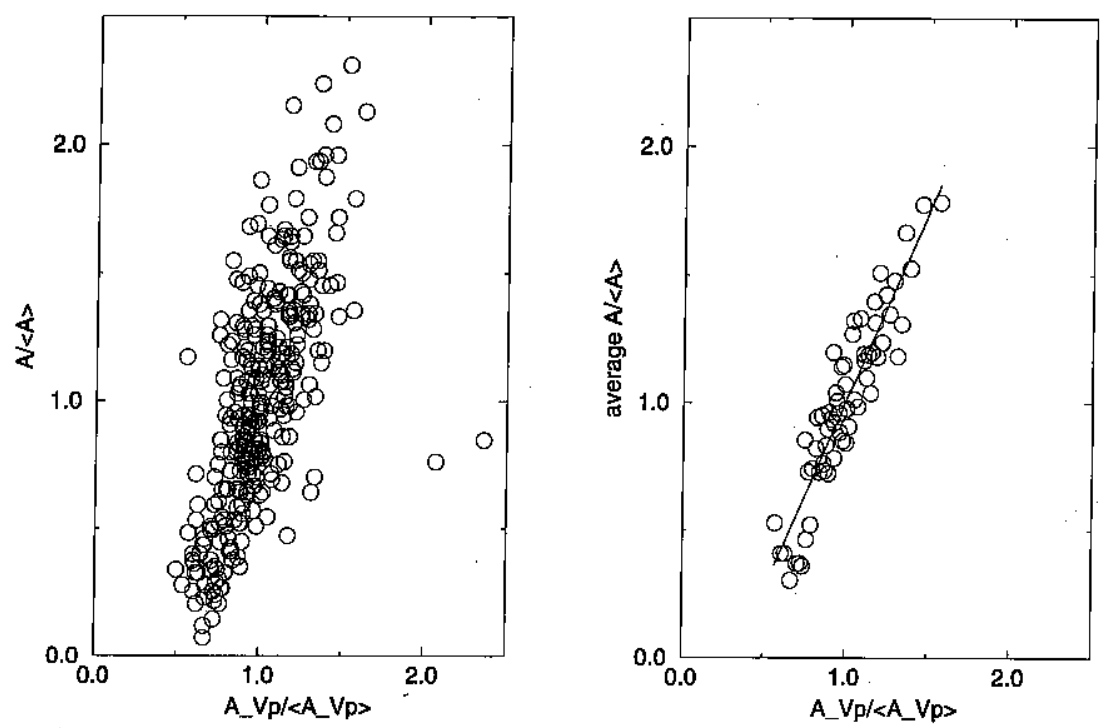

Fig. 3. - Correlation between droplet and Voronoi polygon areas. Left: Scatter plot of areas of droplets, $A$, vs. areas of the corresponding Voronoi cell, $A_{V p}$, each normalized by the respective mean areas, $\langle A\rangle$ and $\left\langle A_{V_{p}}\right\rangle$. Right: Data of plot in left-hand panel, filtered by forming averages of successive sets of five points; also shown is the result of a linear fit, with values of 1.5 and -0.5 for slope and intercept, respectively.

3.2. Droplet Radius Distribution . - From $P_{n}(a, x)$, one computes the distribution $p(x)$ of bubble areas by integrating over $a$ and summing over $n$. This function is found to be virtually independent of $\lambda_{a}, \lambda_{x}$ and $\mu_{2}$ for a reasonable range of these quantities $\left(0 \leq \lambda_{a, x} \leq 0.4\right.$ and $0 \leq \mu_{2} \leq 1$ ). While the weights of partial distributions $p_{n}(x)$ depend on the $p_{n}$ 's and thus on $\mu_{2}$, the total distribution, $p(x)$, remains surprisingly unaffected. Assuming bubbles to adopt the shape of perfects disks, so that $x=\pi r^{2}$, we can compute the radius distribution. The resulting function, $p(x)=\sum_{n} \int \mathrm{d} a P_{n}(a, x)$, essentially represents, for large $x$, a Poisson distribution of droplet area. Under the assumption of circular droplets, of radius $r \sim \sqrt{x}$, this implies a Gaussian tail for the radius distribution.

In Figure 4a, we display the resulting expressions for $p_{n}(r)$ and $p(r)$. A comparison of the ME distribution, $p(r)$, the experimental distributions and the LS form for $p(r)$, shown in Figure $4 \mathrm{~b}$, makes it apparent that both the ME and experimental distributions closely approximate a Gaussian shape, centered at $r \sim \bar{r}$. The experimental distribution is found to be slightly narrower than that given by ME theory, with respective standard deviations of $\sigma=0.235$ for the averaged data in Figure 4 and $\sigma=0.29$ for the best Gaussian fit to the ME distribution. We have occasionally recorded, at early times in off-critical quenches, droplet radius distributions which match the ME shape virtually exactly. In contrast, the LS form is highly asymmetric.

3.3. Spatial Correlations: Screening of Topological Charge - - We now turn to the issue of spatial correlations in cell and bubble patterns. Correlations in the topological charge of NN cells in a disordered pattern are described by the Aboav-Weaire (AW) law [11]. Many disparate physical systems, including cellular patterns, ceramics, soap froths, metallic grains [11], and even Feynman graphs of the $\phi^{3}$ field theory [23], are known to satisfy this law [11]; in contrast, a truely random Voronoi lattice is known [20] to violate it. The AW law has 


\section{Droplet Radius Distribution - Maximum Entropy}

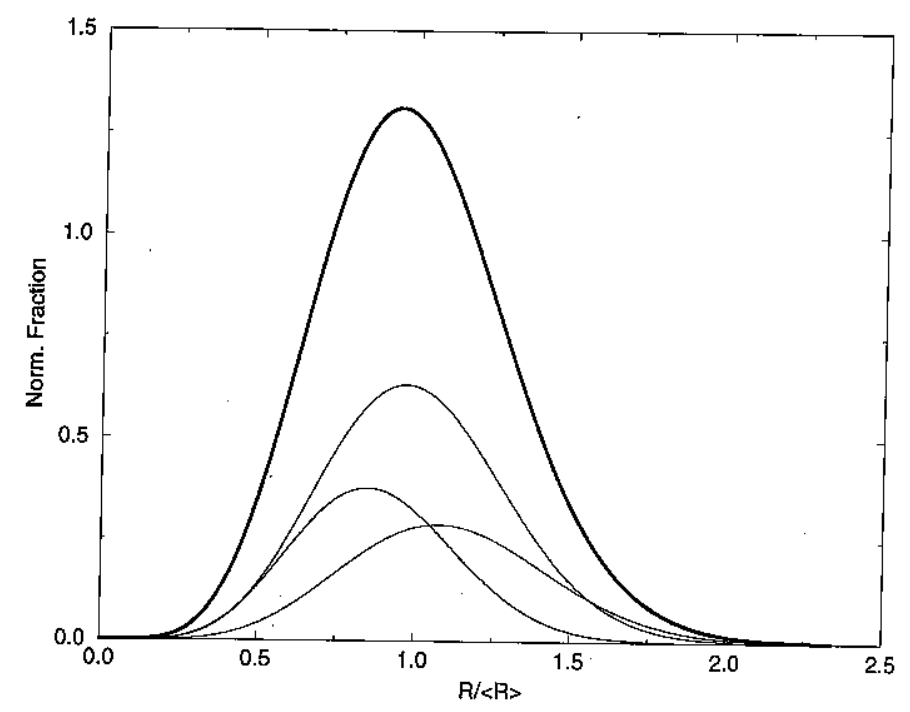

a)

\section{Droplet Radius Distribution}

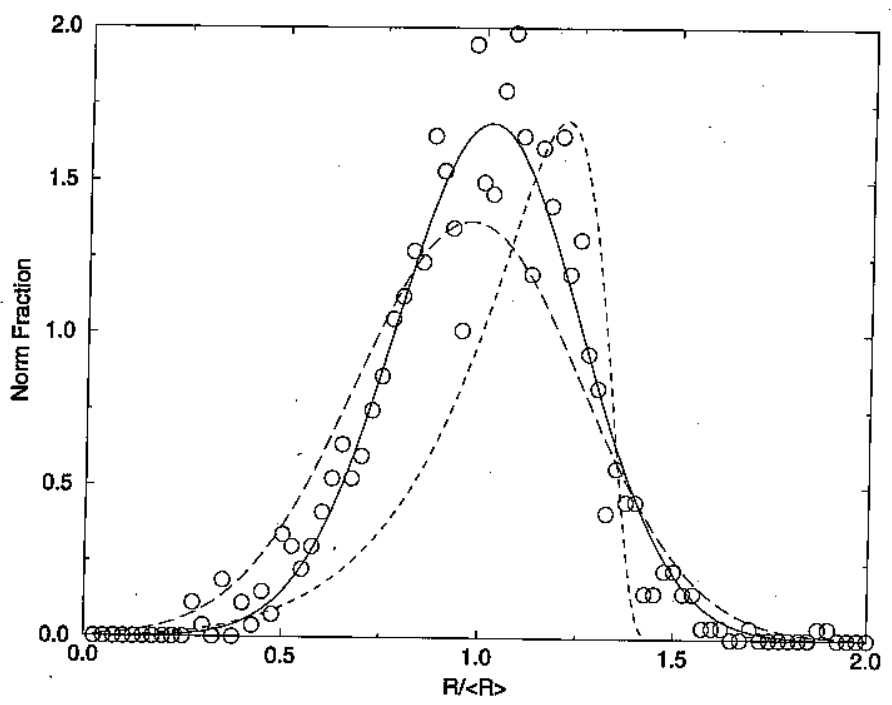

b)

Fig. 4. - Normalized, scaled domain radius distribution. (a): Droplet radius distribution, $p(r)$, from maximum entropy analysis, with $\mu_{2}=0.75$; also shown are the partial distributions, $p_{n}(r), n=4,5,6$. (b): Average of three data sets for the normalized, scaled droplet radius distribution function, recorded at successive times of $17 \mathrm{~min}, 1249 \mathrm{~min}$ and $2946 \mathrm{~min}$ subsequent to an isothermal surface pressure quench [12]. The solid line represents a Gaussian fit, with parameters 1.02 for the mean, 0.24 for the standard deviation and 1.69 for the peak amplitude; the dashed line represent the (best Gaussian fit of the) maximum entropy distribution (with $\mu_{2}=0.75$ ), as discussed in the text; the dotted line represents (the $2 \mathrm{~d}$ version of) the Lifshitz-Slyozov distribution [34]. 
been recently justified from an assumption natural in the framework of ME theory [24].

Defining $C \equiv n-6$ to denote the topological charge of an $n$-sided cell, and $\bar{C}_{\mathrm{NN}}$ to represent the average charge of its $n \mathrm{NN}$ cells, this law states that $C+n \bar{C}_{\mathrm{NN}}=(1-a) C+\mu_{2}$ [25]. Here, $C+n \bar{C}_{\mathrm{NN}}$ is recognized as the net (topological) charge of a "N(earest)N(eighbor)-cluster" of droplets. In view of equation $(3),\langle C\rangle=0$, this parametrization ensures validity of the exact relation $\left\langle C+n \bar{C}_{\mathrm{NN}}\right\rangle=\mu_{2}$ [26]. This sum rule expresses the fact that topological constraints preclude complete charge compensation at the NN level whenever $\mu_{2}>0$. Given that condition, the optimal way to screen topological charge at short range is to minimize the deviations in the net (topological) charge of a NN-cluster from the average. In view of the foregoing, this is achieved by setting the coefficient $1-a=0$, a condition which in fact ensures a vanishing variance $\left\langle\left(n \bar{C}_{\mathrm{NN}}+C-\mu_{2}\right)^{2}\right\rangle=(1-a)^{2} \mu_{2}$.

For most physical systems conforming to the AW law, values of $a \gtrsim 1$ have been reported; the coarsening of droplets of interest here generates patterns which satisfy the AW law throughout the scaling regime, in the narrow range of available $n, 4 \leq n \leq 8$, with a typical value of $a \sim 1.1$. In contrast, for the unphysical Feynman graph pattern, no energetic constraints apply, and one finds $\mu_{2}=10.5$ and $a \approx-1$ [23]. We conclude that the observation of a value of $a \approx 1$ constitutes a strong indication for the importance of charge screening during pattern evolution. Given the linear correlation between droplet areas and topological charge, provided by the Lewis law, the screening of charge implies the suppression of large fluctuations in droplet area about the mean, $\left\langle A_{n}\right\rangle$, and the corresponding patterns are, in that sense, "relaxed".

The condition of minimal variance suggests a heuristic bound on $|1-a|$ : to ensure that the AW law expresses short-range screening, we propose that the variance should not exceed the square of the mean. Equating these two quantities, we obtain the condition $|1-a| \ll \sqrt{\mu_{2}}$. Notice that this argument does not determine the sign of $1-a$. Weaire and Rivier [11] have attempted to account for the fact that $a>1$ on the basis of considering the energetics of inhomogeneities introduced by angular distortions of relaxed cells in polygonal patterns. We prefer to argue on the basis of the connection between droplet area and charge. Specifically, we argue that for $\mu_{2}>0$, it is energetically favorable and hence imperative to ensure efficient screening of large, i.e., larger than average, bubbles. For these, according to the Lewis law, $C>0$, and partial cancellation of $\mu_{2}$ by the correction term $(1-a) C$ in the AW law requires $1-a \leq 0$. Another way to see this is to consider the spatial (not the topological) average of $C+n \bar{C}_{\mathrm{NN}}:\left\langle C+n \bar{C}_{\mathrm{NN}}\right\rangle_{S} \equiv \sum_{n} p_{n} a_{n}\left(C+n \bar{C}_{\mathrm{NN}}\right)=\mu_{2}\left(1+\lambda_{a}(1-a)\right)$. This spatial average of "cluster charge" is clearly smaller if $a>1$ rather than $a<1$.

We now employ the Aboav-Weaire law to examine local spatial correlations in our disordered patterns. Specifically, we focus on the relation between the area $a$ of a Voronoi cell (or the area $x$ of an embedded bubble) and the average area $a^{\prime}$ (or $x^{\prime}$ ) of the NN cells (or bubbles). Measuring $x$ and $x^{\prime}$ in units of $c$, thereby setting both averages to unity, we invoke the Lewis law in the form $x=1+\lambda_{x}(n-6)$ to eliminate $n$ from the Aboav-Weaire law. We readily find

$$
\frac{x^{\prime}}{x}=\frac{1}{x}\left(1+\frac{a(1-x)+\lambda \mu_{2}}{6+(x-1) / \lambda}\right)
$$

An alternative to this topological argument is a geometrical argument, based on the premise that (statistical) area fluctuations (about the average) are completely screened at the NN level: this is ensured by the condition $n x^{\prime}+x=(n+1)$. Once again eliminating $n$ in this expression by employing Lewis's law, we obtain

$$
\frac{x^{\prime}}{x}=\frac{1}{x}\left(1+\frac{(1-x)}{6+(x-1) / \lambda}\right)
$$




\section{Area and Topological Charge Correlations}

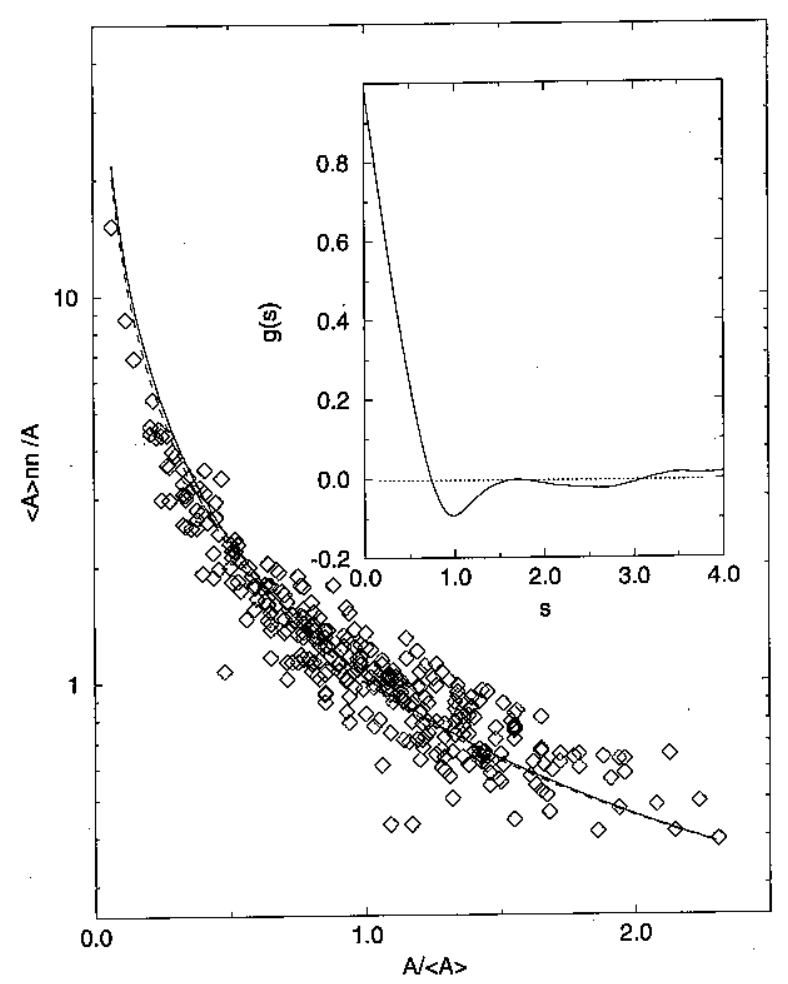

Fig. 5. - Correlations between NN domains. Spatial correlations in the area values assumed by adjacent domains, for the pattern in Figure 1. The solid line represents the model discussed in the text, with parameters $\mu_{2}=0.75, \lambda=0.25$ and $a=1.1$; the dashed line corresponds to the limit of setting $\mu_{2}=0$ and $a=1$, as discussed in the text. Inset: topological charge correlation function. $g(s)=\langle C(r+s) C(r)\rangle_{S} /\langle C(r) C(r)\rangle_{s}$, with $C \equiv n-6$ and distance $s$ measured in units of the average NN centroid distance, $s=\sqrt{\left\langle A_{V p}\right\rangle}$, where $\left\langle A_{V p}\right\rangle$ represents the average Voronoi cell area.

Note that expressions (8) and (9) are identical for $a=1$ and $\lambda \mu_{2}=0$. From this point of view, the observation of values of $a \sim 1$ in physical systems may be taken as an indication that geometric constraints, ensuring short-range screening of area fluctuations, are relevant during pattern evolution. That is, both a topological and a geometrical argument provide a connection between the suppression of large scale inhomogeneities and a value of $a \sim 1$.

In the coarsening experiments, $a \approx 1.1$ and $\lambda \mu_{2} \approx 0.15$, implying that both expressions should be quite close. In Figure 5, we display them along with the experimental points, and find satisfactory agreement. The anti-correlation is apparent, implying that screening of charge and of area fluctuations is in fact relevant here. In fact, it is quite remarkable to find this anticorrelation for bubble areas and not just for the Voronoi cell areas, as would be more intuitive. We note in passing that one can compute the leading correction to LS theory in the small volume fraction limit [14]. The calculation amounts to solving an electrostatics problem with charges of the same sign as the associated bubble growth rate. Thus, charge screening in this problem implies that a growing bubble (which is likely to be large, with $n \geq 6$ ) prefers to be surrounded by shrinking bubbles (likely to be small, with $n \leq 6$ ). This is in accord with the nature of the correlations we find here. 
In the inset of Figure 5 we illustrate the actual charge screening by plotting the experimental charge correlation function $g(s)=\langle C(r+s) C(r)\rangle_{\mathrm{S}} /\langle C(r) C(r)\rangle_{\mathrm{S}}$, with $s$ in units of the average NN centroid distances. The normalization is chosen so that $g(0)=1$ [27]. The NN anticorrelation is clearly seen: one has $g(1) \sim-0.1$ and $g$ rapidly decays to zero at larger distances. The magnitude of $g(1)$ may be understood as follows: using the AW law and neglecting area fluctuations [27], one finds $g(1) \sim\left(6 a+\mu_{2}\right)(1-6\langle 1 / n\rangle) /\left\langle C^{2}\right\rangle \sim-0.14$ with the experimental parameters. A second, small minimum appears at $s \approx 2.5$. While this is expected, given that screening at the $\mathrm{NN}$ level is, in accordance with the Weaire sum rule, impossible, the small amplitude of the second minimum does indicate virtually complete charge compensation at the NN level. That is, topological charges are essentially "associated" into neutral clusters whose size is that of the NN shell.

\section{Discussion}

In the foregoing sections we have extended the maximum entropy description of cellular patterns to droplet patterns, and we have applied this treatment to the analysis of disordered droplet patterns formed during late-stage coarsening in a two-dimensional binary mixture. We have shown that the ME theory accounts well for the virtually Gaussian shape of the scaled droplet radius distribution, as well as for the anti-correlation between areas of adjacent droplets. The latter has been shown to be intimately connected to the screening of topological charge at short distances.

The essential assumption underlying the ME analysis is the predominance of entropic constraints during pattern evolution. That is, for given effective temperature, fixed by prescribing a value for $\mu_{2}$, momentary pattern configurations are selected so as to maximize entropy. Consequently, this formalism does not describe the ordering transition expected to terminate coarsening in a variety of systems with competing short-range attractive and long-range repulsive interactions [5-7], which form (periodically) modulated patterns in equilibrium [9]. The coarsening experiments described here were performed on Langmuir films which exhibit dipolar interactions of long range and are thus thought to belong to this category $[21,28]$. While an ordering transition to a hexagonal lattice of bubbles is thus expected at some late stage, the available evidence suggests that the pertinent cross-over time to equilibrium behavior has not been attained in experiments to date [12, 29]. The present analysis provides strong evidence for a prominent role of entropy maximization in the selection of configurations of coarsening droplet patterns.

The patterns of interest here evolve via configurations which maximize entropy by adopting a local packing arrangement minimizing deviations from a neutral topological state $C \equiv$ $(n-6)=0$. The locally optimal domain packing is stabilized against domain coalescence via repulsive interactions between domains. We propose that it is this stabilization of locally equilibrated pattern configurations during domain coarsening which represents the most notable manifestation of electrostatic interactions in the present system. It appears natural to assume that there are no specific requirements as to the precise distance dependence of the interaction, so long as domain fusion is suppressed. This suggests that, under such conditions, irrespective of spatial dimension, the resulting global correlations favor pattern configurations selected via maximization of entropy.

It is interesting to note that in the very different context of describing quasicrystals, $\mathrm{ME}$ considerations have also been invoked. Given two kinds of elementary tiles (at the proper relative composition), the tiling with maximal configurational entropy is in fact a quasicrystal (for instance a Penrose tiling if the tiles are properly chosen) [30]. This state may thus be viewed as the maximally homogenous mixture of the two tiles, ensuring that a local exchange ("flip-flop") 
of two different vertices is possible everywhere within the pattern. In contrast, in the limit of segregation of the two tiles into patches composed of just one type of tile, i.e. into twinned microcrystals, such tile exchange would be possible only along the crystal boundaries. We see that these microcrystals correspond to a large fluctuation away from the homogeneously mixed state of maximal entropy, and we may thus say that, in the quasicrystal, composition fluctuations are optimally screened [31]. This is in complete analogy to the screening of topological charge and of area fluctuations in the droplet patterns.

Finally, it is an open question whether a von Neumann law applies to the coarsening dynamics of the droplet patterns studied here. This law which is exact for an ideal 2D soap froth [11,32] states that the growth rate of an $n$-sided bubble is $\mathrm{d} A_{n} / \mathrm{d} t \propto(n-6)$; that is, the rate is independent of time and of bubble shape. In the present problem, using the fact that $\langle A\rangle \sim t^{2 / 3}$, and following Rivier's ME treatment for soap bubbles [33], we are lead to conjecture that $\mathrm{d} A_{n} / \mathrm{d} t \propto(n-6) t^{-1 / 3}$. The experimental verification of this relation for individual bubbles, or, more simply, for the average, $\left\langle A_{n}\right\rangle$, of $n$-fold coordinated bubbles, remains an interesting challenge. Numerical simulations could also address this point [34].

\section{References}

[1] Family F., Meakin P., Phys. Rev. A40 (1989) 3836.

[2] Beysens D., Knobler C.M., Phys. Rev. Lett. 57 (1986) 1433;

Viovy J. L., Beysens D., Knobler C. M., Phys. Rev. A 37 (1988) 4965.

[3] For a recent review, see e.g.: Jaeger H.M., Nagel S.R., Science 255 (1992) 1523.

[4] For reviews, see: Gunton J. D., San Miguel M., Sahni P. S., "Phase Transitions and Critical Phenomena", vol.8, C. Domb, J. L. Lebowitz Eds. (Acad. Press, 1983);

Voorhees P. W., J. Stat. Phys. 38 (1985) 231;

Komura S., Phase Transitions 12 (1988) 3.

[5] Bahiana M., Oono Y., Phys. Rev. A41 (1990) 6763.

[6] Chen L.Q., Khachaturyan A.G., Phys. Rev. Lett. 70 (1993) 1477.

[7] Sagui C., Desai R.C., Phys. Rev. Lett. 71 (1993) 3995; Phys. Rev. E49 (1994) 2225.

[8] Seul M., Murray C.A., Science 262 (1993) 558;

Seul M., J. Phys. I France 4 (1994) 319.

[9] Seul M., Andelman D., to be published.

[10] Elliott S. R., "Physics of Amorphous Materials", 2nd. edition (Longman Scientific, Technical, Essex, UK, 1991).

[11] Weaire D., Rivier N., Contemp. Phys. 25 (1984) 59;

Flyvbjerg H., Phys. Rev. 47 (1993) 4037.

[12] Seul M., Morgan N.Y., Sire C., to be published in Phys. Rev. Lett.

[13] Rivier N., J. Phys. Colloq. France 43 (1982) C9-91;

Rivier N., Lissowski R., J. Phys. A15 (1982) L143.

[14] Marder M., Phys. Rev. A36 (1987) 438;

Zheng Q., Gunton J.D., Phys. Rev. A39 (1989) 4848.

[15] Shannon C. E., Bell Systems Techn. J. 27 (1948) 379;

for a concise introduction, see: Papoulis A., "Probability, Random Variables and Stochastic Processes" (McGraw-Hill, NY, 1984) chapt. 15-4.

[16] Preparata F. P., Shamos M. I., "Computational Geometry" (Springer, NY, 1985) chapt. 6.

[17] As noted by Magnasco (Magnasco M.O., Philos. Mag. B65 (1992) 895), relaxation of droplet patterns, by a combination of droplet area adjustment and local translational motion, into an optimized local packing is in fact sufficient to generate a well-defined topology for the droplet pattern. This process likely represents the analog of the equilibration process applied to random Voronoi lattices [11]. 
[18] In general, this rule will be violated for larger area fraction, especially in less homogenous patterns $[1,17]$. In that case, we may obtain a partition by constructing the medium axis transform, or "skeleton" of the majority phase in which droplets are embedded. In this "thinning" construction, the boundary between two cells containing adjacent bubbles is defined by the set of points which are equidistant from the two bubbles in question and are farther from any other bubble; distances are measured to bubble contours (see e.g.: Seul M., Monar L., O'Gorman L., Philos. Mag. B 66 (1992) 471). In the limit of small area fraction this reduces to the Voronoi construction; see also: [16]. For a more general graph-theoretical discussion, see: Magnasco, op.cit. [17]. For simplicity we refer only to Voronoi diagrams in the text.

[19] Lifshitz I.M., Slyozov V.V., J. Phys. Chem. Solids 19 (1961) 35;

Wagner C., Z. Elecktrochem. 65 (1961) 581.

[20] Baekgaard Lauritsen K., Moukarzel C., Herrmann H.J., J. Phys. I France 3 (1993) 1941.

[21] McConnell H.M., Annu. Rev. Phys. Chem. 42 (1991) 171;

McConnell H. M., Rice P. A., Benvegnu D. J., J. Phys. Chem. 94 (1990) 8965.

[22] A further indication of an excluded volume effect is the existence of a minimal distance of approach, $s_{i j}$, between adjacent bubbles: this manifests itself in the form of a quite sharp lower cut-off in the distribution $P\left(s_{i j} /\left\langle s_{i j}\right\rangle\right)$. Based on this cut-off, we estimate an effective area fraction $50 \%-100 \%$ larger than the nominal one. The shape of the distribution is essentially conserved throughout the power law regime of coarsening (Morgan N.Y., Seul M., unpublished).

[23] Godrèche C., Kostov I., Yekutieli Y., Phys. Rev. Lett. 69 (1992) 2674.

[24] Peshkin M.A., Strandburg K.J., Rivier N., Phys. Rev. Lett. 67 (1991) 1803.

[25] The AW law is usually [11] stated in the form $n m(n)=(6-a) n+6 a+\mu_{2}$, where $n$ gives the local coordination and $m$ the average of the coordination number of nearest neighbor droplets; $a$ denotes a phenomenological constant.

[26] This sum rule, stated by Weaire and Rivier [11] in the form $\sum_{n \geq 3} n m(n) p_{n}=36+\mu_{2}$, with $m \equiv \bar{C}_{\mathrm{NN}}$, , may be obtained as follows: $\sum_{i=1}^{N} n_{i} m\left(n_{i}\right) / N=\sum_{i=1}^{N} n_{i}\left(1 / n_{i}\left(\sum_{j \in\{N N\}} n_{j}\right)\right) / N=$ $\sum_{k=1}^{N} n_{k}{ }^{2} / N=\left\langle n^{2}\right\rangle$ which is the desired result, given that $\mu_{2}=\left\langle(n-6)^{2}\right\rangle=\left\langle n^{2}\right\rangle-36$. The sum rule represents the condition that, for finite $\mu_{2}$, strict charge compensation at the nearest neighbor level is topologically impossible. That is, application of the Euler-Poincare condition to the NN shell in the form $(n-6)+\sum_{j \in N N}\left(n_{j}-6\right)=(n-6)+n(m-6)=0$ immediately implies the relation $m=5+6 / n$ which in turn yields $\sum_{n} p_{n} n m(n)=36$. Except for the case of the defect-free hexagonal lattice, with $\mu_{2}=0$, this is in contradiction to the actual sum rule.

[27] The exact form of the charge correlation function at the origin is: $\left\langle C^{2}\right\rangle_{\mathrm{S}}=\sum_{n} p_{n} a_{n}(n-6)^{2}=$ $\mu_{2}+\lambda_{a} \mu_{3}$.

[28] Andelman D., Brochard F., Joanny J. F., J. Chem. Phys. 86 (1987) 3673.

[29] Dipolar interactions may be comparatively more relevant at small area fraction, because geometrical constraints are relaxed in that regime. There are in fact some signs of the existence of clusters of aligned bubbles ("chains") in experiments performed at low area fraction (Morgan N.Y., Seul M., unpublished). In the very dilute limit, coarsening bubbles would evolve independently, and the mean-field LS theory [19] may become applicable and would then describe the droplet ensemble. In contrast, even in the dilute limit, the Voronoi pattern of centroids should be well described by maximum entropy considerations.

[30] Elser V., unpublished;

Henley C. L., J. Phys. A21 (1988) 1649.

[31] Sire C., Gratias D., "Statics and Dynamics of Alloy Phase Transformations", P.E.A. Turchi and A. Gonis Eds. (Plenum Press, NY, 1994).

[32] For a three-dimensional generalization of von Neumann law see, Sire C., Phys. Rev. Lett. 72 (1994) 420.

[33] Rivier N., Physica 32D (1986) 129.

[34] Rogers T.M., Desai R.C., Phys. Rev. B39 (1989) 11956. 